\title{
Front Matter: Volume 7017
}

, "Front Matter: Volume 7017," Proc. SPIE 7017, Modeling, Systems Engineering, and Project Management for Astronomy III, 701701 (17 July 2008); doi: $10.1117 / 12.804988$

Event: SPIE Astronomical Telescopes + Instrumentation, 2008, Marseille, France 


\title{
PROCEEDINGS OF SPIE
}

\section{Modeling, Systems Engineering, and Project Management for Astronomy III}

\author{
George Z. Angeli \\ Martin J. Cullum \\ Editors
}

26-28 June 2008

Marseille, France

Sponsored by

SPIE

SPIE Europe

Cooperating Organizations

AAS-American Astronomical Society (USA) • ASJ-Astronomical Society of Japan (Japan)

AURA-Association of Universities for Research in Astronomy, Inc. (USA) • Ball Aerospace \& Technologies

Corporation (USA) • CNRS-Centre National de la Recherche Scientifique (France) • EAS—European

Astronomical Society (Switzerland) • ESO_European Southern Observatory (Germany) • IAU-International Astronomical Union (France) • INSU-Institut National des Sciences de I'Univers (France) • LAM-Laboratoire d'Astrophysique de Marseille (France) - MPE-Max-Planck-Institut für extraterrestrische Physik (Germany) NAOJ-National Astronomical Observatory of Japan (Japan) • NASA—NASA Goddard Space Flight Center (USA) • Northrop Grumman Corporation (USA) • OAMP-Observatoire Astronomique de Marseille Provence (France) OPTICON-Optical Infrared Coordination Network (United Kingdom) • RadioNet-Advanced Radio Astronomy in Europe (United Kingdom) • Royal Astronomical Society (United Kingdom) • Science \& Technology Facilities Council (United Kingdom) • SFO-Société Française d'Optique (France) Competitiveness Cluster: POPsud-Pôle Optique \& Photonique (France) • Optitec Sud (France)

Published by

SPIE

Volume 7017

Proceedings of SPIE, 0277-786X, v. 7017

SPIE is an international society advancing an interdisciplinary approach to the science and application of light. 
The papers included in this volume were part of the technical conference cited on the cover and title page. Papers were selected and subject to review by the editors and conference program committee. Some conference presentations may not be available for publication. The papers published in these proceedings reflect the work and thoughts of the authors and are published herein as submitted. The publisher is not responsible for the validity of the information or for any outcomes resulting from reliance thereon.

Please use the following format to cite material from this book:

Author(s), "Title of Paper," in Modeling, Systems Engineering, and Project Management for Astronomy III, edited by George Z. Angeli, Martin J. Cullum, Proceedings of SPIE Vol. 7017 (SPIE, Bellingham, WA, 2008) Article CID Number.

ISSN 0277-786X

ISBN 9780819472274

Published by

SPIE

P.O. Box 10, Bellingham, Washington 98227-0010 USA

Telephone +1 3606763290 (Pacific Time) · Fax +1 3606471445

SPIE.org

Copyright (c) 2008, Society of Photo-Optical Instrumentation Engineers

Copying of material in this book for internal or personal use, or for the internal or personal use of specific clients, beyond the fair use provisions granted by the U.S. Copyright Law is authorized by SPIE subject to payment of copying fees. The Transactional Reporting Service base fee for this volume is $\$ 18.00$ per article (or portion thereof), which should be paid directly to the Copyright Clearance Center (CCC), 222 Rosewood Drive, Danvers, MA 01923. Payment may also be made electronically through CCC Online at copyright.com. Other copying for republication, resale, advertising or promotion, or any form of systematic or multiple reproduction of any material in this book is prohibited except with permission in writing from the publisher. The CCC fee code is 0277-786X/08/\$18.00.

Printed in the United States of America.

Publication of record for individual papers is online in the SPIE Digital Library.

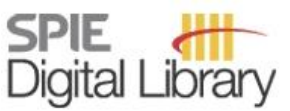

SPIEDigitalLibrary.org

Paper Numbering: Proceedings of SPIE follow an e-First publication model, with papers published first online and then in print and on CD-ROM. Papers are published as they are submitted and meet publication criteria. A unique, consistent, permanent citation identifier (CID) number is assigned to each article at the time of the first publication. Utilization of CIDs allows articles to be fully citable as soon they are published online, and connects the same identifier to all online, print, and electronic versions of the publication. SPIE uses a six-digit CID article numbering system in which:

- The first four digits correspond to the SPIE volume number.

- The last two digits indicate publication order within the volume using a Base 36 numbering system employing both numerals and letters. These two-number sets start with $00,01,02,03,04,05$, 06, 07, 08, 09, OA, OB ... 0Z, followed by 10-1Z, 20-2Z, etc.

The CID number appears on each page of the manuscript. The complete citation is used on the first page, and an abbreviated version on subsequent pages. Numbers in the index correspond to the last two digits of the six-digit CID number. 


\title{
Contents
}

\author{
xi Conference Committee \\ xiii Introduction \\ xv High redshift galaxy surveys (Plenary Paper) [7016-500] \\ M. Iye, National Astronomical Observatory (Japan)
}

\section{SESSION 1 SYSTEMS ENGINEERING FOR GROUND BASED PROJECTS}

701702 ATST systems engineering: project update and lessons learned [7017-01]

R. P. Hubbard, National Solar Observatory (United States)

701704 Systems engineering for the preliminary design of the Thirty Meter Telescope [7017-03] G. Z. Angeli, Thirty Meter Telescope Observatory (United States); S. Roberts, Herzberg Institute of Astrophysics (Canada); K. Vogiatzis, Thirty Meter Telescope Observatory (United States)

701705 An integrated systems approach for the modeling of infrared instruments for the next generation telescopes [7017-04]

H. Schnetler, W. D. Taylor, United Kingdom Astronomy Technology Ctr., Royal Observatory (United Kingdom)

701706 Efficiency evaluation of proposed EAGLE target acquisition systems [7017-05] W. D. Taylor, C. Evans, H. Schnetler, United Kingdom Astronomy Technology Ctr., Royal Observatory Edinburgh (United Kingdom)

701707 Management of optical interfaces in the VLT KMOS instrument [7017-06]

P. Rees, United Kingdom Astronomy Technology Ctr., Royal Observatory Edinburgh (United Kingdom); R. Content, M. Dubbeldam, Durham Univ. (United Kingdom); I. Lewis, Oxford Astrophysics (United Kingdom); S. Rolt, Durham Univ. (United Kingdom); S. Todd, United Kingdom Astronomy Technology Ctr., Royal Observatory Edinburgh (United Kingdom); I. Tosh, Rutherford Appleton Lab. (United Kingdom)

\section{SESSION 2 SYSTEMS ENGINEERING FOR SPACE PROJECTS}

701708 System engineering and management in a large and diverse multinational consortium [7017-07]

D. Wright, B. O'Sullivan, J. Thatcher, I. Renouf, Astrium, Ltd. (United Kingdom); G. Wright, M. Wells, A. Glasse, UK Astronomy Technology Ctr., Royal Observatory (United Kingdom); U. Grozinger, Max-Planck-Institut für Astronomie (Germany); J. Sykes, Univ. of Leicester (United Kingdom); D. Smith, P. Eccleston, B. Shaughnessy, Rutherford Appleton Lab. (United Kingdom) 
7017 OA James Webb Space Telescope Mid-Infrared Instrument cooler systems engineering [7017-09]

K. Banks, NASA Goddard Space Flight Ctr. (United States); M. Larson, Jet Propulsion Lab. (United States); C. Aymergen, SGT, Inc. (United States); B. Zhang, Jet Propulsion Lab. (United States)

7017 OB Unique integration and test philosophy for the Wide-field Infrared Survey Explorer flight system [7017-10] J. F. Howard, M. Shannon, N. Taylor, Ball Aerospace \& Technologies Corp. (United States); F. Liu, M. Abid, I. Heinrichsen, Jet Propulsion Lab. (United States)

7017 OC Alignment of two mirror astronomical telescopes (the astigmatic component) [7017-11] T. Schmid, The College of Optics and Photonics, Univ. of Central Florida (United States); K. Thompson, Optical Research Associates (United States); J. Rolland, The College of Optics and Photonics, Univ. of Central Florida (United States)

7017 OD LOTIS at completion of Collimator integration [7017-12]

R. M. Bell, G. C. Robins, C. Eugeni, G. Cuzner, S. B. Hutchison, Lockheed Martin Space Systems Co. (United States); S. H. Baily, B. Ceurden, J. Hagen, K. Kenagy, H. M. Martin, M. Tuell, M. Ward, S. C. West, Steward Observatory, Univ. of Arizona (United States)

\section{SESSION 3 PROJECT MANAGEMENT I}

7017 OG Organisation and management of the ALMA project (Invited Paper) [7017-15] H. Rykaczewski, European Organisation for Astronomical Research in the Southern Hemisphere (Germany)

$7017 \mathrm{OH}$ Managing a big ground-based astronomy project: the Thirty Meter Telescope (TMT) project (Invited Paper) [7017-16]

G. H. Sanders, Thirty Meter Telescope Project (United States)

\section{SESSION 4 PROJECT MANAGEMENT II}

7017 OK Cost scaling of finely segmented filled aperture large telescope observatories: a TMT study [7017-19]

J. Oram, Triad Project Management Services (United States); D. Goodman, G. Sanders, TMT Observatory Corp. (United States)

7017 OL The SPHERE exoplanet imager: a new management paradigm? [7017-20] F. Wildi, Observatoire de Genève (Switzerland); J.-L. Beuzit, Lab. d'Astrophysique de Grenoble (France); M. Feldt, Max Planck Institut für Astronomie (Germany); D. Mouillet, Lab. d'Astrophysique de Grenoble (France); K. Dohlen, Lab. d'Astrophysique de Marseille (France); P. Puget, Lab. d'Astrophysique de Grenoble (France) 
7017 OM Development of the Wide-field Infrared Survey Explorer (WISE) mission [7017-21] F. Liu, Jet Propulsion Lab. (United States); R. Cutri, Infrared Processing and Analysis Ctr. (United States); G. Greanias, V. Duval, P. Eisenhardt, Jet Propulsion Lab. (United States); J. Elwell, Space Dynamics Lab. (United States); I. Heinrichsen, Jet Propulsion Lab. (United States); J. Howard, Ball Aerospace \& Technologies Corp. (United States); W. Irace, A. Mainzer, Jet Propulsion Lab. (United States); A. Razzaghi, NASA Goddard Space Flight Ctr. (United States); D. Royer, Jet Propulsion Lab. (United States); E. L. Wright, Univ. of California, Los Angeles (United States)

7017 ON Management in a U.S./European partnership: the MIRI instrument on JWST [7017-22] P. A. Driggers, SGT, Inc. (United States); P. Barela, Jet Propulsion Lab. (United States)

\section{SESSION 5 MODELING OF WIND AND THERMAL SEEING}

$701700 \quad$ Wind turbulence structure inside telescope enclosures [7017-23]

M. Quattri, European Southern Observatory (Germany); R. Haase, Gesellschaft für Bemessungforschung mbH (Germany); M. Barreto Cabrera, Instituto de Astrofísica de Canarias (Spain); E. Friend Monasterio, ITER, Pol. Industrial de Granadilla (Spain); R. Codina, Univ. Politecnica de Catalunya (Spain); M. Gonzalez Hernandez, Polytechnic Univ. of Madrid (Spain); M. Reyes, Instituto de Astrofísica de Canarias (Spain)

7017 OP Initial design factors and airflow analysis for the LSST enclosure [7017-24] D. R. Neill, J. D. Barr, V. L. Krabbendam, NOAO/LSST (United States); K. K. Khankari, A. S. Watave, Y. Krishna, ANSYS, Inc. (United States)

$7017 \mathrm{OQ}$ Advances in aerothermal modeling for TMT [7017-25] K. Vogiatzis, TMT Observatory Corp. (United States)

7017 OR Dome and mirror seeing estimates for the Thirty Meter Telescope [7017-26] J. S. Pazder, National Research Council Canada, Herzberg Institute of Astrophysics (Canada); K. Vogiatzis, G. Z. Angeli, Thirty Meter Telescope Observatory (United States)

\section{SESSION 6 PERFORMANCE MODELING I}

7017 OS An integrated model of the European Extremely Large Telescope [7017-27]

T. E. Andersen, A. Enmark, P. Linde, M. Owner-Petersen, A. Sjöström, Lund Observatory (Sweden); F. Koch, M. Müller, L. Noethe, B. Sedghi, European Southern Observatory (Germany)

7017 OT Analysis of Normalized Point Source Sensitivity as a performance metric for the Thirty Meter Telescope [7017-28]

B.-J. Seo, C. Nissly, Jet Propulsion Lab. (United States) and California Institute of Technology (United States); G. Angeli, B. Ellerbroek, J. Nelson, Thirty Meter Telescope Observatory (United States); N. Sigrist, M. Troy, Jet Propulsion Lab. (United States) and California Institute of Technology (United States) 
7017 OU High-resolution optical modeling of the Thirty Meter Telescope for systematic performance trades [7017-30]

C. Nissly, B.-J. Seo, M. Troy, Jet Propulsion Lab. (United States) and California Institute of Technology (United States); G. Angeli, Thirty Meter Telescope Observatory (United States) and California Institute of Technology (United States); J. Angione, Jet Propulsion Lab. (United States) and California Institute of Technology (United States); I. Crossfield, Univ. of California, Los Angeles (United States); B. Ellerbroek, L. Gilles, Thirty Meter Telescope Observatory (United States) and California Institute of Technology (United States); N. Sigrist, Jet Propulsion Lab. (United States) and California Institute of Technology (United States)

7017 OV Monte Carlo simulation framework for TMT [7017-29]

K. Vogiatzis, G. Z. Angeli, TMT Observatory Corp. (United States)

7017 OW Dynamic analysis of TMT [7017-31]

D. G. MacMynowski, California Institute of Technology (United States); C. Blaurock, Nightsky Systems, Inc. (United States); G. Z. Angeli, Thirty Meter Telescope Observatory (United States)

\section{SESSION 7 PERFORMANCE MODELING II}

7017 OX Optical modeling activities for NASA's James Webb Space Telescope (JWST): Part V. Operational alignment updates [7017-32] J. M. Howard, K. Q. Ha, R. Shiri, J. S. Smith, G. Mosier, D. Muheim, NASA Goddard Space Flight Ctr. (United States)

7017 OY The ESA-developed NIRSpec performance simulator [7017-33] M. te Plate, B. Dorner, European Space Agency (Netherlands); P.-A. Frugier, Commissariat à l'Energie Atomique (France)

$70170 Z$ The JWST/NIRSpec instrument performance simulator [7017-34]

L. Piqueras, P.-J. Legay, E. Legros, P. Ferruit, A. Pecontal, Univ. de Lyon, Observatoire de Lyon, CNRS (France); X. Gnata, P. Mosner, ASTRIUM GmbH (Germany)

$701710 \quad$ Numerical simulation of the VLT/MUSE instrument [7017-35]

A. Jarno, R. Bacon, P. Ferruit, A. Pécontal-Rousset, Univ. de Lyon, Observatoire de Lyon, CNRS (France) and Ctr. de Recherche Astrophysique de Lyon, Ecole Normale Supérieure de Lyon (France)

701711 Integrated modeling for the GPi flexure sensitive structure [7017-36]

D. Erickson, S. Roberts, J. S. Pazder, J. M. Fletcher, National Research Council Canada, Herzberg Institute of Astrophysics (Canada)

\section{SESSION 8 CONTROLS SYSTEM MODELING}

701713 Simulation of the Planck-HFI thermal control system [7017-38]

C. Leroy, IAS, Univ. Paris Sud, CNRS (France); M. Maisonneuve, CESR, Univ. Paul Sabatier, CNRS (France); M. Piat, APC, Univ. Paris VII, CNRS (France); J.-F. Trouilhet, LATT, Univ. Paul Sabatier, CNRS (France); D. Pearson, JPL (United States); C. Camier, S. Guibert, APC, Univ. Paris VII, CNRS (France) 
701715 Analysis of TMT primary mirror control-structure interaction [7017-41]

D. G. MacMynowski, California Institute of Technology (United States); P. M. Thompson, Systems Technology, Inc. (United States); M. J. Sirota, Thirty Meter Telescope Observatory (United States)

\section{SESSION 9 STRUCTURAL AND THERMAL MODELING}

701716 Thermal performance prediction of the TMT optics [7017-43]

M. Cho, GSMT Program Office, National Optical Astronomy Observatory (United States);

A. Corredor, S. Pootrakul, Univ. of Arizona (United States); K. Vogiatzis, G. Angeli, TMT

Project Office, California Institute of Technology (United States)

701717 X-shooter near infra-red spectrograph cryogenic design [7017-44]

R. Roelfsema, NOVA-ASTRON (Netherlands); P. Albers, Radboud Univ. Nijmegen

(Netherlands); J.-L. Lizon, ESO (Germany); P. van Dael, Radboud Univ. Nijmegen

(Netherlands); E. Elswijk, NOVA-ASTRON (Netherlands); P. Groot, Radboud Univ. Nijmegen

(Netherlands); H. Hanenburg, J. Kragt, R. Navarro, N. Tromp, NOVA-ASTRON (Netherlands);

G. Wulterkens, Radboud Univ. Nijmegen (Netherlands)

701718 Modeling temperature-induced surface distortions in the MMTO 6.5 meter primary mirror [7017-45]

T. E. Pickering, MMT Observatory (United States)

701719 Advanced Technology Solar Telescope lower enclosure thermal system [7017-46]

L. Phelps, M. Warner, National Solar Observatory (United States)

7017 1A Spatial Nyquist fidelity method for structural models of opto-mechanical systems [7017-74] D. Howell, The Aerospace Corp. (United States); O. de Weck, D. W. Miller, Massachusetts Institute of Technology (United States)

\section{POSTER SESSION: PROJECT MANAGEMENT}

7017 1B The ALMA Band 3 (84-116 GHz) receiver production plan [7017-47]

K. Yeung, S. Claude, D. Loop, National Research Council of Canada (Canada)

7017 1C Thirty Meter Telescope cost estimating process [7017-48]

J. Oram, Triad Project Management Services (United States); D. Goodman, G. Sanders, TMT Observatory Corp. (United States)

7017 IF Tonantzintla's Observatory Astronomy Teaching Laboratory project [7017-51]

F. Garfias, A. Bernal, L. A. Martínez, L. Sánchez, H. Hernández, R. Langarica, A. Iriarte, J. H. Peña, S. Tinoco, F. Ángeles, Instituto de Astronomía, Univ. Nacional Autónoma de México (Mexico) 
7017 IG FRACTAL Systems \& Project suite: engineering tools for improving development and operation of the systems [7017-53]

A. Pérez-Calpena, E. Mujica-Alvarez, J. Osinde-Lopez, M. García-Vargas, FRACTAL SLNE. (Spain)

$7017 \mathrm{IH} \quad$ The image quality error budget for the VST telescope [7017-54]

P. Schipani, F. Perrotta, INAF-Osservatorio Astronomico di Capodimonte (Italy)

701711 Exploring model based engineering for large telescopes: getting started with descriptive models [7017-55]

R. Karban, M. Zamparelli, B. Bauvir, B. Koehler, L. Noethe, A. Balestra, European Southern Observatory (Germany)

$70171 \mathrm{~J}$ System definition of the James Webb Space Telescope (JWST) Integrated Science Instrument Module (ISIM) [7017-56]

R. Lundquist, NASA Goddard Space Flight Ctr. (United States); C. Aymergen, SGT, Inc. (United States); J. Van Campen, Mantech (United States); J. Abell, SGT, Inc. (United States); M. Smith, NASA Goddard Space Flight Ctr. (United States); P. Driggers, SGT, Inc. (United States)

\section{POSTER SESSION: MODELING}

7017 1K Enola Gay: an integrated modelling optical toolbox applied to a wide-field telescope [7017-59]

P. Schipani, F. Perrotta, INAF-Osservatorio Astronomico di Capodimonte (Italy)

7017 1L Simulation model of FAST focus cabin for pointing accuracy analysis [7017-60] J. Sun, R. Nan, W. Zhu, National Astronomical Observatories (China); H. J. Kärcher, MT Mechatronics GmbH (Germany); H. Li, National Astronomical Observatories (China)

$70171 \mathrm{M}$ System architectural design of multiwavelength data mining [7017-61]

Y. Zhang, Y. Zhao, National Astronomical Observatories (China); H. Zheng, North China Electric Power Univ. (China)

7017 iN Integrated thermal disturbance analysis of optical system of astronomical telescope [7017-62]

D. Yang, Z. Jiang, X. Li, National Astronomical Observatories, Nanjing Institute of Astronomical Optics and Technology (China)

7017 IP Modeling large deformable mirrors [7017-64]

R. Heimsten, T. Andersen, M. Owner-Petersen, Lund Observatory (Sweden)

7017 1Q Advanced 2D spectroscopic predicted data [7017-65]

P. Bristow, F. Kerber, ESO (Germany); M. R. Rosa, ESA (Germany)

7017 IR Filling the gap between FEM and control simulations in telescope projects [7017-66]

F. Perrotta, P. Schipani, INAF-Osservatorio Astronomico di Capodimonte (Italy) 
7017 is Earthquake safety system design for a telescope primary mirror [7017-67]

F. Perrotta, INAF-Osservatorio Astronomico di Capodimonte (Italy)

7017 1T Implementation of differential wavefront sampling in optical alignment of pupil-segmented telescope systems [7017-68]

H. Lee, Univ. of Oxford (United Kingdom); G. B. Dalton, Univ. of Oxford (United Kingdom) and Rutherford Appleton Lab. (United Kingdom); I. A. J. Tosh, Rutherford Appleton Lab. (United Kingdom); S.-W. Kim, Space Optics Lab., Yonsei Univ. (South Korea)

$70171 \mathrm{~V}$ Aspects of error budgeting for coronagraphic differential imaging: effects of dust and cosmetic errors [7017-70]

K. Dohlen, Lab. d'Astrophysique de Marseille, CNRS, Univ. de Provence (France)

7017 IW Autonomous high dynamic range phase unwrapping [7017-71]

S. Bikkannavar, Jet Propulsion Lab. (United States)

$70171 \mathrm{X}$ Analysis of the spectral surface radiance by using the surface BRDF and the atmospheric transmission effects [7017-72]

J.-H. Choi, T.-K. Kim, Chung-Ang Univ. (South Korea)

7017 1Y Finite element analysis of TMT vibrations transmitted through Telescope-Enclosure-Soil Interaction [7017-73]

G. Shagal, ACURA, Univ. of Toronto (Canada); D. G. MacMynowski, California Institute of Technology (United States); K. Vogiatzis, Thirty Meter Telescope Observatory (United States)

$701712 \quad$ Modeling of a large deformable mirror for future E-ELT [7017-75]

R. Gasmi, GEPI, Observatoire de Paris, CNRS, Univ. Paris Diderot (France); J. C. Sinquin, Cilas (France); P. Jagourel, J. L. Dournaux, GEPI, Observatoire de Paris, CNRS, Univ. Paris Diderot (France); D. Le Bihan, Onera (France); F. Hammer, GEPI, Observatoire de Paris, CNRS, Univ. Paris Diderot (France)

Author Index 
Downloaded From: https://www.spiedigitallibrary.org/conference-proceedings-of-spie on 26 Apr 2023

Terms of Use: https://www.spiedigitallibrary.org/terms-of-use 


\title{
Conference Committee
}

\author{
Symposium Chairs
}

Mark C. Clampin, NASA Goddard Space Flight Center (United States)

Alan F. M. Moorwood, European Southern Observatory (Germany)

Symposium Cochairs

Masanori lye, National Astronomical Observatory of Japan (Japan)

Douglas A. Simons, Gemini Observatory (United States)

Conference Chairs

George Z. Angeli, Thirty Meter Telescope Observatory (United States)

Martin J. Cullum, European Southern Observatory (Germany)

Program Committee

Torben E. Andersen, Lund University, Lund Observatory (Sweden)

Mark C. Clampin, NASA Goddard Space Flight Center (United States)

Xiangqun Cui, LAMOST Project (China)

Philippe Dierickx, European Southern Observatory (Germany)

Eric R. Hansen, National Solar Observatory (United States)

David G. Hunter, James Webb Space Telescope, Space Telescope Science Institute (United States)

Matt W. Johns, Observatories of the Carnegie Institution of Washington (United States)

David W. Miller, Massachusetts Institute of Technology (United States)

Hermine Schnetler, United Kingdom Astronomy Technology Centre, The Royal Observatory, Edinburgh (United Kingdom)

Donald W. Sweeney, LSST Corporation (United States)

\section{Session Chairs}

1 Systems Engineering for Ground Based Projects

Eric R. Hansen, National Solar Observatory (United States)

$2 \quad$ Systems Engineering for Space Projects

Hermine Schnetler, United Kingdom Astronomy Technology Centre, The Royal Observatory, Edinburgh (United Kingdom) 
3 Project Management I

George Z. Angeli, Thirty Meter Telescope Observatory (United States)

Martin J. Cullum, European Southern Observatory (Germany)

4 Project Management II

Matt W. Johns, Observatories of the Carnegie Institution of Washington (United States)

$5 \quad$ Modeling of Wind and Thermal Seeing

David G. Hunter, James Webb Space Telescope, Space Telescope Science Institute (United States)

6 Performance Modeling I

George Z. Angeli, Thirty Meter Telescope Observatory (United States)

7 Performance Modeling II

Torben E. Andersen, Lund University, Lund Observatory (Sweden)

8 Controls System Modeling

Hermine Schnetler, United Kingdom Astronomy Technology Centre, The Royal Observatory, Edinburgh (United Kingdom)

$9 \quad$ Structural and Thermal Modeling

Donald W. Sweeney, LSST Corporation (United States) 


\section{Introduction}

Project management and systems engineering activities are crucial to ensure the successful delivery of complex, high technology projects. While project management is responsible for the timely delivery of the project within budget, systems engineering is responsible to deliver a product that will be fit for use and will fulfill the users' expectations. An essential element of systems engineering is the creation of realistic systems models that can be used to validate critical assumptions and verify the performance of the proposed system solutions.

As astronomical projects grow in scope and budget as well as becoming geographically more distributed, programmatic and technical management constitutes significant challenges. Project management tools and practices are aimed to mitigate technical, schedule, and cost risks, while facilitating "design to cost" process.

The systems engineering process begins with the establishment of the top-level requirements and continues through the design, manufacturing, and implementation phases, as well as through operation, maintenance, and eventually disposal. By facilitating a consistent and well-documented system design as well as a sound integration process, the systems engineer ensures that the project will be both scientifically rewarding and technically feasible.

System modeling is essential to validate performance allocations by proving that the entire system will achieve the high-level objectives if each system component meets its own requirements. Modeling is also vital for verifying the performance of the design developed. Simulation results are critical parts of system integration and test plans, while they also help in understanding unexpected behavior during implementation and commissioning.

This conference arches from traditional systems engineering fields, such as requirement definition, analysis, and flow-down, system architectural design, and performance analysis, to the emerging fields of end-to-end and integrated modeling. It also covers closely related areas of program management, such as financial modeling, parametric cost estimate, risk analysis, technology roadmaps, and life-cycle considerations.

The conference on Modeling and Systems Engineering was included for the first time in the SPIE symposium on Astronomical Telescopes and Instrumentation in Glasgow in June 2004. It attracted considerable interest then, and this interest continued at the meeting held in Orlando in May 2006 when project management was added to the scope of the conference. In this 2008 Symposium in Marseille, particular emphasis was placed on the synergy between ground and space projects, and this was reflected in many of the presentations. 
A particular success was the session on the management of large ground-based and space projects which included five invited talks from speakers from NASA, ESA, ALMA, TNT, and the E-ELT.

George Z. Angeli

Martin J. Cullum 


\title{
High redshift galaxy surveys
}

\author{
Masanori Iye \\ National Astronomical Observatory, Mitaka, Tokyo, 181-8588 Japan
}

\begin{abstract}
A brief overview on the current status of the census of the early universe population is given. Observational surveys of high redshift galaxies provide direct opportunities to witness the cosmic dawn and to have better understanding of how and when infant galaxies evolve into mature ones. It is a much more astronomical approach in contrast to the physical approach of to study the spatial fluctuation of cosmic microwave radiation. Recent findings in these two areas greatly advanced our understanding of the early Universe. I will describe the basic properties of several target objects we are looking for and the concrete methods astronomers are using to discover those objects in early Universe. My talk starts with Lyman $\alpha$ emitters and Lyman break galaxies, then introduces a clever approach to use gravitational lensing effect of clusters of galaxies to detect distant faint galaxies behind the clusters. Finally I will touch on the status and prospects of surveys for quasars and gamma-ray bursts.
\end{abstract}

Keywords: gamma ray burst, high redshift, Lyman $\alpha$ emitter, Lyman break galaxy, quasar, survey

*m.iye@nao.ac.jp; phone 8142234 3520; fax 81422343527

\section{INTRODUCTION}

Since the discovery of the expansion of the Universe by Edwin Hubble in 1929, astronomers with ever more powerful telescopes surveyed the sky to find more and more distant galaxies. By studying distant galaxies, one can look back the early history of the Universe. Partridge and Peebles ${ }^{1}$, in their classical 1967 paper, predicted the properties of primordial galaxies and pointed out that these galaxies with redshifted Lyman $\alpha$ emission are the targets observational astronomers should look for. Many attempts followed using $4 \mathrm{~m}$ class telescopes for next three decades. This was, however, not an easy $\operatorname{task}^{2}$.

Astronomers of this decade developed various techniques to isolate distant objects; narrow band imaging surveys for Lyman $\alpha$ emitting galaxies ${ }^{3-28}$, multi-band photometric surveys for Lyman break galaxies ${ }^{29-38}$, searches for amplified images of gravitationally lensed galaxies ${ }^{39-47}$, quasars ${ }^{48-54}$ and studies of sporadic gamma ray bursts ${ }^{55-57}$ in high redshift galaxies. Galaxies up to redshift $z=6.96^{18}$ were spectroscopically confirmed and there are additional candidate galaxies that appear to be at redshift $\mathrm{z}>7^{34-37,41,44,45}$.

The current picture of the big bang Universe indicates that the expanding universe cooled rapidly to form neutral hydrogen from protons and electrons at 380,000 years after the big bang. This is the epoch when the photons are decoupled from the matter. The density fluctuation of the dark matter and the matter grew by gravitational interaction and it is conceived that the first generation of stars were born at around 200 million years after the big bang. Initial set of formed stars contained wide range of mass spectrum. The absence of metal elements in the primordial gas helped to form massive stars. Due to the strong UV radiation from those newly formed massive hot stars, the surrounding intergalactic matter was gradually re-ionized. A kind of "Global Warming of the Universe". When and how these re-ionization process took place is not observationally clarified yet but WMAP5 results ${ }^{59}$ suggest $\mathrm{z} \sim 11$ if the re-ionization was an instantaneous event. It is more likely that the cosmic re-ionization could have taken place in an extended period sometime during $6<\mathrm{z}<17$.

Detailed observations deep into the era beyond $z=7$ is, therefore, crucial. Some of the recent number counts of galaxies at $5.7<\mathrm{z}<7$ indicate significant decrease in the number density of Lyman $\alpha$ emitting galaxies $^{16-18}$, which could either be 
due to the evolution of galaxies possibly through merging processes or due to the increasing fraction of neutral hydrogen blocking Lyman $\alpha$ emitting galaxies at high redshift.

I will describe the target population of galaxies in the early Universe and the technique astronomers are employing to find those objects together with some recent results.

\section{NARROW BAND SURVEY FOR LYMAN A EMITTERS}

What are Lyman $\alpha$ emitters, that are often abbreviated ash LAEs? They are thought to be star-forming young galaxies with star formation rate from 1 to 10 solar mass per year. Hot massive stars produce strong UV radiation field and ionize the interstellar gas. The ionized hydrogen recombines and cools by emitting a Lyman a photon to settle down to the lowest ground level. The amount of stars produced in these galaxies is not yet very large as the usual continuum radiation from stars is not necessarily conspicuous. The spectra of LAEs are therefore characterized by strong Lyman- $\alpha$ emission line as shown in Fig.1.

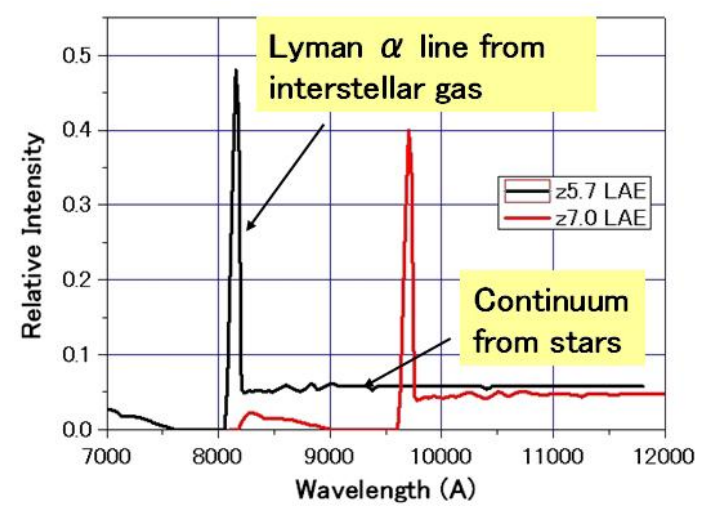

Fig. 1. Typical spectra of Lyman- $\alpha$ emitters showing conspicuous Lyman $\alpha$ emission lines.

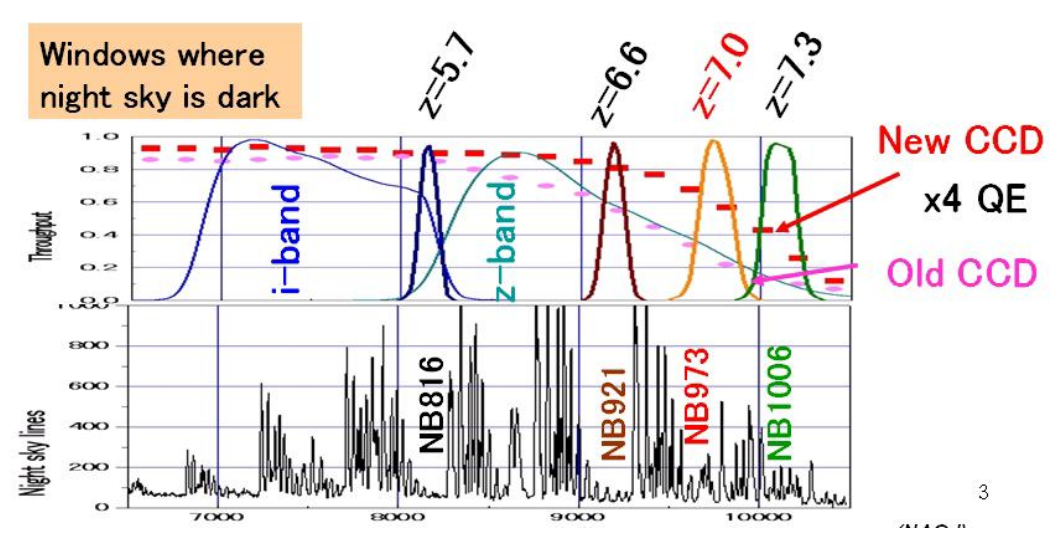

Fig. 2. OH night sky emission bands (lower panel) show a few gaps, which astronomers use as dark windows to study deep into the Universe. Narrow band filters whose transmission are matched to these dark windows are used to sample LAEs at $z=5.7$ (NB816), $z=6.6$ (NB921) and $z=7.0$ (NB973). The current CCD sensitivity falls rapidly toward $1000 \mathrm{~nm}$ but recently developed high-resistivity, red-sensitive CCDs open a possibility to extend the accessible redshift limit up to $\mathrm{z}=7.3$. 
How to find those LAEs? It would be natural to catch the Lyman $\alpha$ emission line signal from these galaxies. Since these objects are so faint, one has to consider the properties of the sky background, actually foreground radiation from the Earth's atmosphere. The night sky glows ever brighter at longer wavelength. In the wavelength region below 1 micron, where Si-CCDs are sensitive, the night sky spectrum shows strong bands of $\mathrm{OH}$ emission lines as shown in the lowest panel of Fig.2. The gaps between these $\mathrm{OH}$ bands are nice dark windows to probe deep space.

Astronomers use narrow band filters whose transmittance bands are matched to one of these gaps to pick up light only in this gap to detect LAEs whose redshifted Lyman a emission enters in this gap. LAEs at appropriate redshift range are expected to show up brighter in the narrow band image than other broad band images. The narrow band (NB) survey is therefore trying to slice the universe in a narrow range of redshift. There are several such gaps, for instance, the narrow band filter NB816 that has the central wavelength at $816 \mathrm{~nm}$ is suitable for isolating LAEs at redshift 5.7, NB921nm for redshift 6.6, etc. The most distant LAE at redshift 7.0 confirmed to date was also discovered using the narrow band imaging survey using a filter centered at $973 \mathrm{~nm}$. The sensitivity of current CCDs falls rapidly toward 1 micron but recent advent of red sensitive CCDs with thicker depletion layer will extend this redshift limit slightly up to about 7.3.

Let me talk on our discovery of the most distant galaxy. The red blob in the left panel of Fig. 3 shows the most distant galaxy, IOK- $1^{18}$. This LAE was discovered among the 41,533 objects in the Subaru Deep Field through the narrow band filter NB973 for a total of 15 hours with SuprimeCam ${ }^{58}$. All the objects were cross identified in images taken in other filters and only five photometric candidates for $z=7$ LAEs, which are visible only in this narrow band filter, were isolated (cf. Fig.4). Astronomers have a privilege to name their newly found objects and we took a liberty of naming them taking the initials of three main contributes to this survey, IOK-1 to IOK-5.

We have to be, however, careful as there are several types of possible contaminants in these 5-sigma photometric candidates. First, since the narrow band imaging observation was made 1-2 year after other broad band observations, some of the candidates may well be variable objects like AGNs or galaxies where supernovae added extra light when narrow band observation was made. Possibility for emission line objects at lower redshift is a common concern. To our surprise, simple statistics cautions us that there might be one or two 5 sigma noises as well, since there are millions of independent 2 arcsec apertures one can sample in the SuprimeCam field. Spectroscopic follow-up revealed that only one object, the brightest IOK-1, is a real LAE at redshift 6.96, with the characteristic asymmetric line profile as shown in the right panel of Fig.3.

Table 1 shows the top 10 list of high redshift galaxies with spectroscopic redshift measurement, to the best of my knowledge. You may notice that 9 out of 10 were discovered by Subaru/SuprimeCam survey in the single Subaru Deep Field. This is because Subaru/SuprimeCam enables observation of large survey volume with significant depth. Hubble Ultra Deep Field imaging survey with ACS probes much deeper than ground based observations, but has a much smaller survey volume. The wide field surveys to pick up scarce bright population and narrow field deep surveys to study fainter populations, are complementary to each other.

Subaru Deep Field surveys yielded several dozens of LAE candidates both at redshift 5.7 and 6.6 and about half of them are already confirmed spectroscopically to be LAEs. With this fair sample, one can derive the luminosity function of LAEs. The left panel of Fig.5 shows the UV continuum luminosity functions of LAEs at redshift 5.7 and 6.6 which are, more or less, identical. On the other hand, the right panel shows the Lyman a luminosity functions. We can see that the brighter population of LAEs at redshift 6.6 is significantly less abundant as compared to those at redshift 5.7.

This can be explained if the neutral hydrogen fraction of the intergalactic matter is increasing from redshift 5.7 to 6.6 , as the neutral hydrogen selectively absorbs and scatters the Lyman $\alpha$ photons but not for UV continuum. The Ly- $\alpha$ luminosity functions, the UV luminosity functions, and the distribution of equivalent width of the LAEs can be reconciled with the presence of Pop III massive star formation followed by PoP II star formation to power Ly- $\alpha$ emission $^{60}$. Of course, the scarcity in LAEs at high redshift could also be due to the evolutionary history of those galaxies building from tiny proto galaxies. Cosmic variance could be another factor, if not significant to this level. 

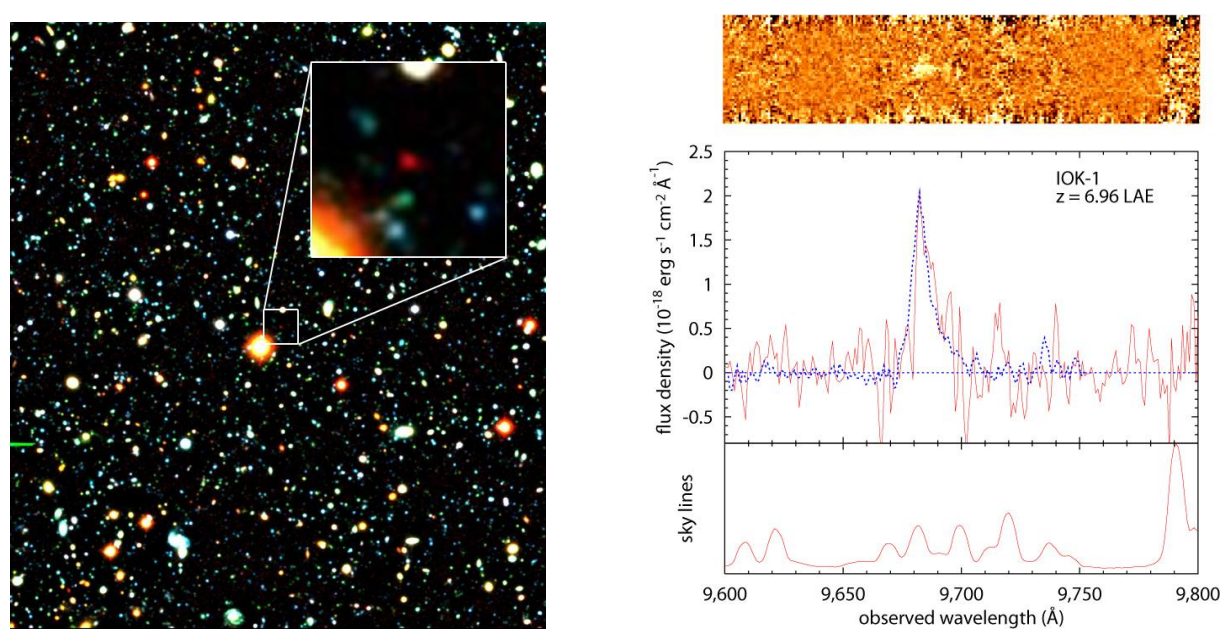

Fig. 3. (Left) The most distant galaxy IOK-1 is shown as a red blob in the inlet panel. (Right) Spectrum of IOK-1 showing the characteristic Lyman $\alpha$ emission line with an asymmetric profile at $968 \mathrm{~nm}$ indicating its redshift 6.96 (Right panel reproduced from Iye et al., $2006^{18}$ ).

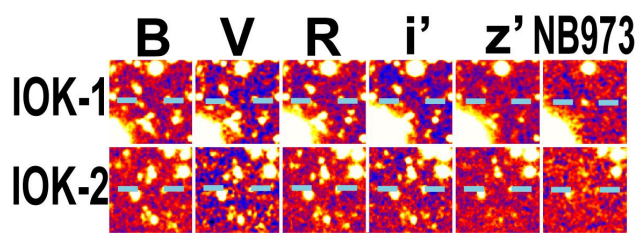

Fig. 4. Post stamp images of the NB973 objects IOK-1 and IOK-2. The latter was confirmed to be a 5-sigma noise (Edited from Ota et al., $2008^{22}$ ).

Table 1: The most distant galaxies with measured redshift (as of June 6, 2008).

\begin{tabular}{lllllll}
\hline Rank & ID & Coordinates & $z$ & Gyr & Paper & Published date \\
\hline \hline 1 & IOK-1 & J132359.8+272456 & 6.964 & 12.88 & Iye et al. & Sep. 14, 2006 \\
2 & SDF ID1004 & J132522.3+273520 & 6.597 & 12.82 & Taniguchi et al. & Feb, 25, 2005 \\
3 & SDF ID1018 & J132520.4+273459 & 6.596 & 12.82 & Kashikawa et al. & Apr. 25, 2006 \\
4 & SDF ID1030 & J132357.1+272448 & 6.589 & 12.82 & Kashikawa et al. & Apr. 25, 2006 \\
5 & SDF ID1007 & J132432.5+271647 & 6.580 & 12.82 & Taniguchi et al. & Feb. 25, 2005 \\
6 & SDF ID1008 & J132518.8+273043 & 6.578 & 12.82 & Taniguchi et al. & Feb. 25, 2005 \\
6 & SDF ID1001 & J132418.3+271455 & 6.578 & 12.82 & Kodaira et al. & Apr. 25, 2003 \\
$8^{*}$ & HCM-6A & J023954.7-013332 & 6.560 & 12.82 & Hu et al. & Apr. 1, 2002 \\
9 & SDF ID1059 & J132432.9+273124 & 6.557 & 12.82 & Kashikawa et al. & Apr. 25, 2006 \\
10 & SDF ID1003 & J132408.3+271543 & 6.554 & 12.82 & Taniguchi et al. & Feb.25, 2005 \\
\hline
\end{tabular}

Table 1. Top 10 list of the most distant galaxies. .

In order to identify LAEs at $\mathrm{z}>7$, quite a few projects to make narrow band imaging surveys with near infrared cameras are under way or planned ${ }^{23-28}$. The field of view of infrared cameras is still considerably smaller than that of, e.g., SuprimeCam and the increasing night sky background make the infrared imaging survey very challenging if the LAE luminosity function is further declining from $\mathrm{z}=6.6$ to further redshift. 


\section{TWO COLOR DIAGNOSIS FOR LYMAN BREAK GALAXIES}

Another population of galaxies searched for in the early Universe is called Lyman Break Galaxies, abbreviated as LBGs. LBGs are thought to be fairly massive galaxies with evolved stellar population. Stellar continuum is much stronger than LAEs. Lyman $\alpha$ emission is less conspicuous as compared with LAEs. The spectra of these galaxies show characteristic discontinuity at the blue side of Lyman $\alpha$ line caused by the intrinsic stellar atmospheric absorption and by the Intergalactic neutral hydrogen absorption. These galaxies, therefore, are visible at bands redward of Lyman $\alpha$ line but are not visible at bands blueward of the Lyman $\alpha$ line. One can select out LBG candidates at $\mathrm{z}=6$ by $\mathrm{i}$-band dropouts, $\mathrm{z}=7$ by $z$ '-band dropout, and $z=9$ by J-band dropouts.

Here again, one have to be careful for possible contaminants. Galactic T-dwarfs dwell in the similar region in two color diagram. One may be able to reject T-dwarfs by their point source images if the image quality is superb. Variable objects and 5 sigma noises are the common problems for this survey as wall.

Hubble ACS and NICMOS imaging at Hubble Ultra Deep Field and GOODS field was used to identify faint z-dropouts at around $z=7.3$ and about 8 candidates were isolated. but similar attempt for $\mathrm{J}$ dropout didn't yield a candidate ${ }^{37}$. Another group reported finding of $10 \mathrm{z}$-dropouts and $2 \mathrm{~J}_{\text {-dropouts }}{ }^{46}$.

Unfortunately, many of these objects do not show strong Lyman $\alpha$ emission and spectroscopic confirmation of their genuine redshift is difficult.

\section{SURVEY FOR STRONGLY LENSED GALAXIES}

Let me turn to genius survey projects using the gravitational lensing effect of a massive cluster of galaxies to magnify and brighten the background faint galaxies. Cluster of galaxies are largest telescopes in the Universe with diameter about

1Mpc. They are nice telescopes for astronomers. You do not need to ask for funding agencies for construction budget and you do not need to ask engineers to design and build them. They are in situ and free of charge to use. Of course there are some drawbacks. You cannot point them to your favorite targets. Wavefront aberrations are bazaar. Although the images produced by cluster lensing are peculiarly deformed and enlarged, the largest advantage is the fact some of the lensed images are brightened considerably and when multiply lensed images are available they can be used to check for the consistency of their reconstructed source image.

Appropriate modeling of the gravitational field of the cluster enables the prediction of the location of critical lines for assumed source redshift slice where the magnification becomes infinity. Observers can look for lensed object along these critical lines and there are in fact several candidate galaxies found in this way ${ }^{39-47}$. For instance, a survey for strongly lensed LAEs in 9 clusters yielded six candidates ${ }^{44}$. If any of these candidates are real, the number density of faint population of galaxies is much larger than previously considered and may well explain the necessary amount of reionizing source.

Fig.6 shows a promising z-dropout candidate at redshift 7.6 found behind the cluster Abel 1689 recently ${ }^{45}$. Photometric results indicate better match to a galaxy at $z=7.6$, however, here again the possibility of galaxy at $z=1.7$ is hard to rule out just from imaging.

\section{QUASARS AND GAMMA RAY BURSTERS}

The last objects I am going to introduce are point sources, quasars and gamma ray bursts (GRBs), in the early Universe. The survey technique used to isolate high redshift quasar candidates is similar to that used for LBGs. Objects that match the expected spectral energy distribution of high redshift quasars are surveyed in the two color diagram or even a multidimension color manifold. Sloan Digital Sky Survey with its enormous data base is a nice test bed to apply this 
approach. Many quasars beyond redshift 6 were found in this way ${ }^{48-52}$. The most distant quasar to date is J1148+5251 at $6.42^{51}$. Gunn-Peterson test of quasars up to redshift 6 indicated strongly that the cosmic re-ionization ended by redshift 6 .
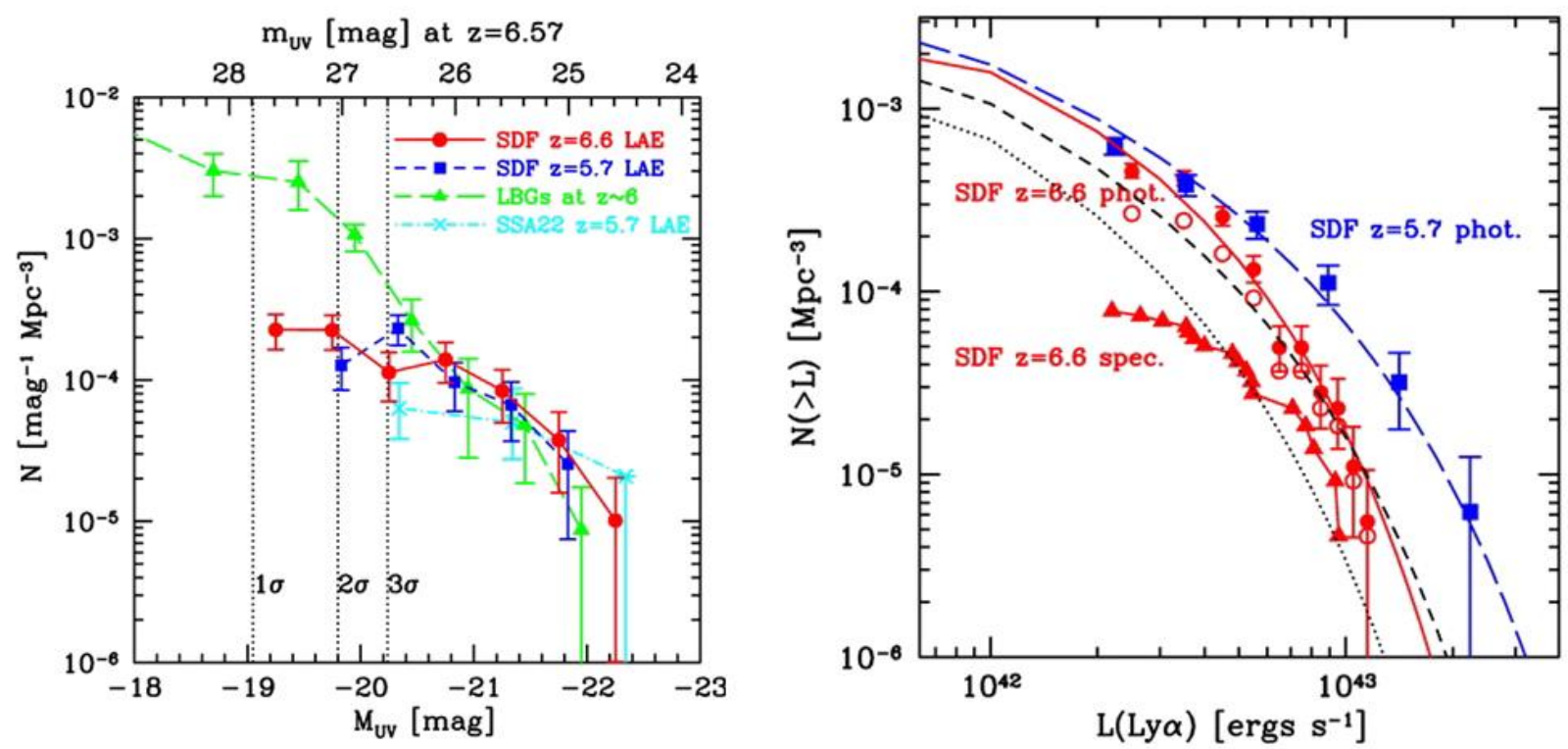

Fig. 5. (Left panel) UV continuum luminosity function of LAEs at $z=5.7$ (blue) and $z=6.6$ (red) which are more or less identical. (Right panel) Lyman $\alpha$ luminosity functions of LAEs at $\mathrm{z}=5.7$ (blue) and $\mathrm{z}=6.6$ (red). Note that the significant decrease in Lyman- $\alpha$ luminosity function at its bright end (Edited from Kashikawa et al., 2006 ${ }^{17}$ ).

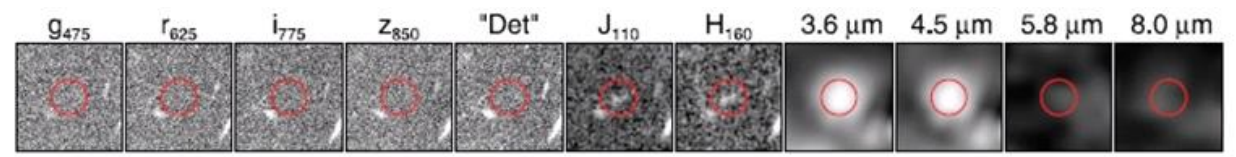

\section{Critical line for $z=7.6$}
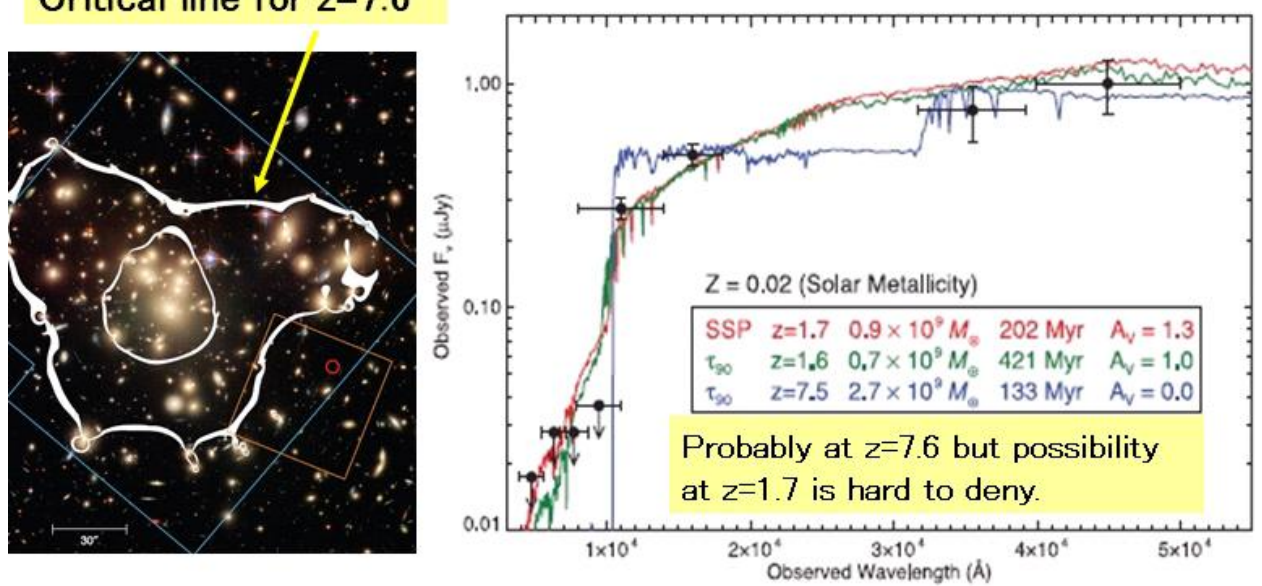

Fig.6. Lyman break galaxy candidate at $\mathrm{z} \sim 7.6$ discovered behind the lensing cluster A1689 (Edited from Bradley et al. $2008^{45}$ ). 
The advent of the real time alert system of gamma ray burst increased the chance of optical and infrared astronomers to make prompt observations of these rapidly declining bursts. The most distant GRB observed to date is GRB050904 at $\mathrm{z}=6.3^{55}$. GRBs at high redshift can be useful tools to probe the cosmic re-ionization through its Lyman- $\alpha$ damping wing $^{56}$.

GRB has a much simpler featureless continuum than the quasar spectra which has broad emission lines superposed on the non-thermal continuum. GRBs are, in a way, better probes to study the re-ionization history. Both quasars and GRBs are point sources, the advent of laser guide star adaptive optics makes the observation of fainter objects feasible and we expect many such observations if the observatories pay efforts for timely follow-up spectroscopy of long burst GRBs. GRBs may provide a new way to study even higher-redshift galaxies and first generation of stars.

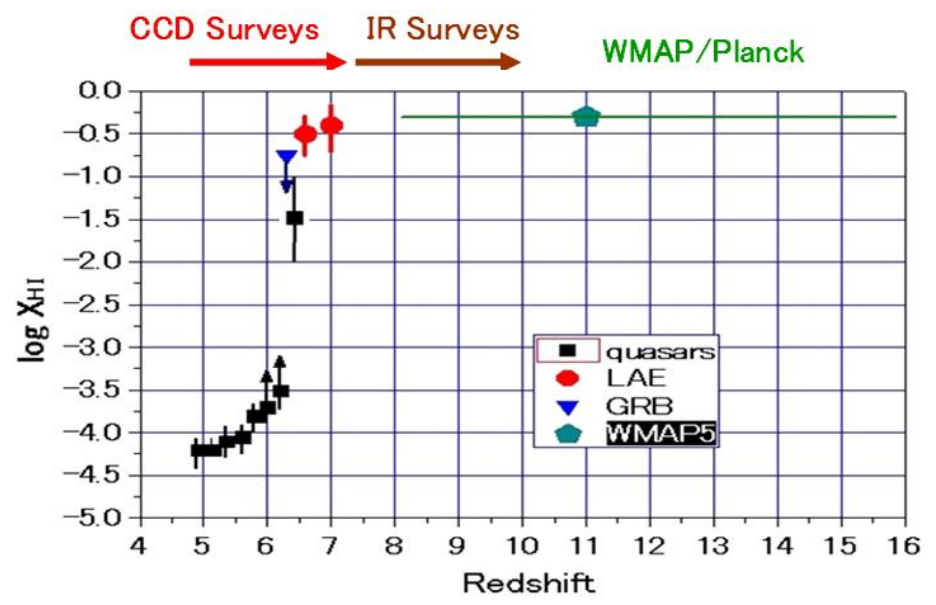

Fig. 7. Neutral hydrogen fraction of intergalactic matter as derived from Gunn-Peterson tests of $\mathrm{z}>5$ quasars (black squares), damped Lyman $-\alpha$ wing profile (blue triangle), and Lyman $\alpha$ luminosity function (red circles). Also plotted is the WMAP 5 year result, which predict $\mathrm{z}=11$ for instantaneous re-ionization. Note, however, that WMAP cannot constrain when re-ionization started and how long it took to complete.

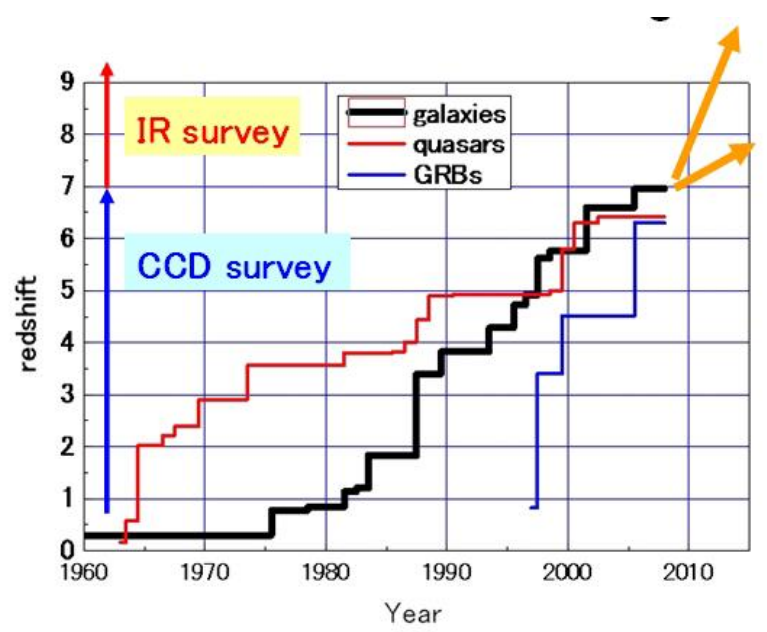

Fig.8. Growth history of largest redshift objects. Note that GRBs are catching up quickly (Based on Tanvir \& Jakobsson, $2007^{57}$ ) 
Fig.7 shows the increase of the fraction of neutral hydrogen as measured from Gunn-Peterson test ${ }^{54}$ of quasars up to redshift 6.42 on the left hand. Our results from redshift 6.6 and 7.0 LAE is shown in red and an upper limit from redshift 6.3 GRB is shown in blue triangle. WMAP5 polarization study concludes that the cosmic re-ionization, if it took place instantaneously, would be at redshift around $11^{59}$ However, WMAP results alone cannot pin down when the cosmic reionization started and how log did it take to finish. Planck satellite my give more clue in 5 years time. Surveys for galaxies beyond redshift 7 up to 11 is, therefore, extremely important to elucidate what happened actually in this period and for that we need NIR deep surveys.

My last slide (Fig. 8) shows the annual growth of the records of highest redshift objects ${ }^{57}$. The discovery of our $\mathrm{z}=6.96$ galaxy was announced on Sep.14, 2006, 648 days ago. Simple statistical argument ${ }^{61}$ predicts that new record will come in, at $95 \%$ confidence level, at earliest in 17 days from today and at latest in 69 years. I am confident, however, that we do not need to wait so long as lots of new surveys are under way using near infrared cameras. Besides, observations of GRBs are catching up quickly, and considering the availability of innovated LGSAO, I would rather predict GRB will soon take over this race.

\section{REFERENCES}

[1] Partridge, R.B. \& Peebles, P. J. E., “Are young galaxies visible?”, Astrophys. J., 147, 868-886 (1967)

[2] Pritchet, C.J., "The search for primeval galaxies", Publ. Astron. Soc. Pacific, 106, 1052-1067 (1994)

[3] Hu, E. M. \& McMahon, R. G., "Detection of Lyman- $\alpha$-emitting galaxies at redshift 4.55", Nature 382, 231-233 (1996).

[4] Cowie, L. L. \& Hu, E. M., "High-z Ly- $\alpha$ emitters. I. A blank-field search for objects near redshift $\mathrm{z}=3.4$ in and around the Hubble Deep Field and the Hawaii Deep Field SSA22", Astron. J. 115, 1319-1328 (1998).

[5] Loeb, A. \& Rybicki, G. B., "Scattered Ly- $\alpha$ radiation around sources before cosmological re-ionization", Astrophys. J. 524, 527-535 (1999).

[6] Hu, E. M. et al., "A redshift z = 6.56 galaxy behind the cluster Abell 370", Astrophys. J. 568, L75-L79 (2002).

[7] Kodaira, K. et al., "The discovery of two Lyman $\alpha$ emitters beyond redshift 6 in the Subaru Deep Field", Publ. Astron. Soc. Jpn 55, L17-L21 (2003).

[8] Santos, M. R., Ellis, R. S., Kneib, J.-P., Richard, J. \& Kuijken, K., "The abundance of low luminosity Ly- $\alpha$ emitters at high redshift", Astrophys. J. 606, 683-701 (2004).

[9] $\mathrm{Hu}$, E. M. et al., "The luminosity function of Ly- $\alpha$ emitters at redshift z 5.7”, Astron. J. 127, 563-575 (2004).

[10] Kurk, J. D. et al., "A Lyman $\alpha$ emitter at $z=6.5$ found with slitless spectroscopy", Astron. Astrophys. 422, L13-L17 (2004).

[11] Rhoads, J. E. et al., "A luminous Ly- $\alpha$-emitting galaxy at redshift $\mathrm{z}=6.535$ : Discovery and spectroscopic confirmation”, Astrophys. J. 611, 59-67 (2004).

[12] Stanway, E. R. et al., "Three Ly- $\alpha$ emitters at $\mathrm{z} \sim 6$ : Early GMOS/Gemini data from the GLARE project", Astrophys. J. 604, L13-L16 (2004).

[13] Malhotra, S. \& Rhoads, J. E., "Luminosity functions of Ly- $\alpha$ emitters at redshifts $z=6.5$ and $z=5.7$ : Evidence against re-ionization at z $<6.5$ ", Astrophys. J. 617, L5-L8 (2004).

[14] Nagao, T. et al., "A strong Ly- $\alpha$ emitter at $z=6.33$ in the Subaru Deep Field selected as an i'-dropout”, Astrophys. J. 613, L9-L12 (2004).

[15] Taniguchi, Y. et al., "The SUBARU Deep Field Project: Lyman $\alpha$ emitters at a redshift of 6.6", Publ. Astron. Soc. Jpn 57, 165-182 (2005)

[16] Shimasaku, K., et al, "Ly alpha Emitters at z=5.7 in the Subaru Deep Field", PASJ, 58, 313-334, (2006)

[17] Kashikawa, N., et al., "The End of the Re-ionization Epoch Probed by Ly-alpha Emitters at $\mathrm{z}=6.5$ in the Subaru Deep Field", Astrophys.J, 648, 7, (2006)

[18] Iye, M. et al., "A Galaxy at a redshift 6.96", Nature, 443, 186-188 (2006).

[19] Hu, E.M. \& Cowie, L.L., "High-redshift galaxy populations", Nature, 440, 1145-1150 (2006)

[20] Ouchi, M. et al., "Exploring the Cosmic Dawn with Subaru Telescope", ASP Conf. Series, 379, 47-54 (2007)

[21] Stark, D.P. et al., "An empirically calibrated model for interpreting the evolution of galaxies during the re-ionization era", Astrophys.J., 668, 627-642 (2007) 
[22] Ota, K. et al., "re-ionization and galaxy evolution probed by z=7 Ly- $\alpha$ emitters", Astrophys.J., 677, 12-26 (2008)

[23] Horton, et al., "DAzLE: The Dark Ages z(redshift) Lyman- $\alpha$ Explorer”, SPIE, 5492, 1022, (2004)

[24] Ichikawa,T. et al., "MOIRCS Deep Survey II. Clustering properties of K-band selected galaxies in GOODS-North region", PASJ, 59, 1081-1094 (2007)

[25] Cuby, et al., "A narrow-band search for Ly- $\alpha$ emitting galaxies at z=8.8”, Astron \& Astrophys., 461, 911-916 (2007)

[26] Nilsson, K.K. et al., "Narrow-band surveys for very high redshift Lyman- $\alpha$ emitters", Astron. \& Astrophys. 474, 385-392 (2007)

[27] Geach, J.E., et al., "HiZELS: a high redshift survey of H-alpha emitters. I: the cosmic star-formation rate and clustering at z=2.23", arXiv:0805.2861 (2008)

[28] Smail,I. et al., "The HiZELS Survey", http://astro.dur.ac.uk/ irs/HiZELS/

[29] Stanway, E. R., Bunker, A. J. \& McMahon, R. G., "Lyman break galaxies and the star formation rate of the Universe at z $\sim 6 "$, Mon. Not. R. Astron. Soc. 342, 439-445 (2003).

[30] Bouwens, R., Broadhurst, T. \& Illingworth, G., "Cloning dropouts: Implications for galaxy evolution at high redshift", Astrophys. J. 593, 640-660 (2003).

[31] Bouwens, R. J. et al., "Galaxies at z 7-8: z850-dropouts in the Hubble Ultra Deep Field", Astrophys. J. 616, L79L82 (2004).

[32] Bunker, A. J., Stanway, E. R., Ellis, R. S. \& McMahon, R. G., "The star formation rate of the Universe at $\mathrm{z} \sim 6$ from the Hubble Ultra-Deep Field", Mon. Not. R. Astron. Soc. 355, 374-384 (2004).

[33] Stanway, E. R. et al., "Hubble Space Telescope imaging and Keck spectroscopy of z $~ 6$ i-band dropout galaxies in the Advanced Camera for Surveys GOODS fields", Astrophys. J. 607, 704-720 (2004).

[34] Yan, H. \& Windhorst, R. A., "Candidates of $\mathrm{z} \approx 5.5-7$ galaxies in the Hubble Space Telescope Ultra Deep Field", Astrophys. J. 612, L93-L96 (2004).

[35] Bouwens, R. \& Illingworth, G., "Rapid Evolution in the most luminous galaxies during the first 900 million years", Nature 443, 189-192 (2006)

[36] Henry, A.L., et al., “A Lyman break galaxy candidate at z 9”, arXiv:0805.1228v1 (2008), NICMOS/IRAC photometric survey for J dropouts.

[37] Bouwens, R. et al., "z 7-10 galaxies in the HUDF and GOODS fields, and their UV luminosity functions", arXiv0803.0548v2 (2008)

[38] Oesch, P. A., "The UDF05 follow-up of the HUDF: II. Constraints on re-ionization from z-dropout galaxies", arXiv:0804.4874v1 (2008)

[39] Kneib, J.-P., Ellis, R. S., Santos, M. R. \& Richard, J., “A probable $\mathrm{z} \sim 7$ galaxy strongly lensed by the rich cluster A2218: Exploring the Dark Ages”, Astrophys. J. 607, 697-703 (2004).

[40] Egami, E. et al., "Spitzer and Hubble Space Telescope constraints on the physical properties of the $z \sim 7$ galaxy strongly lensed by A2218”, Astrophys. J. 618, L5-L8 (2005). A galaxy lensed by A2218 possibly at redshift z 6.66.8 .

[41] Willis, J. P. \& Courbin, F., “A deep, narrow J-band search for protogalactic Ly- $\alpha$ emission at redshifts z $\sim 9$ ”, Mon. Not. R. Astron. Soc. 357, 1348-1356 (2005).

[42] Yan, H. et al., "Rest-frame ultraviolet-to-optical properties of galaxies at $\mathrm{z} \sim 6$ and $\mathrm{z} \sim 5$ in the Hubble Ultra Deep Field: From Hubble to Spitzer”, Astrophys. J. 634, 109-127 (2005)

[43] Chary, R.-R., Stern, D. \& Eisenhardt, P., "Spitzer constraints on the $\mathrm{z}=6.56$ galaxy lensed by Abell 370", Astrophys. J. 635, L5-L8 (2005)

[44] Stark, D.P. et al., "A Keck survey for gravitationally-lensed Lyman- $\alpha$ emitters in the redshift range $8.5<\mathrm{z}<10.4$ : New constraints on the contribution of low luminosity sources to cosmic re-ionization", Astrophys.J. 663, 10-28 (2007)

[45] Bradley, L.D., et al., "Discovery of a very bright strongly lensed galaxy candidate at z 7.6”, Astrophys. J. 678, 647654 (2008)

[46] Richard, J. et al., “A Hubble \& Spitzer Space Telescope Survey of Gravitationally-lensed galaxies: Further evidence for a significant population of low luminosity galaxies beyond redshift seven”, arXis:0803.4391v2

[47] Yan, H.J., et al., "Search for Very High-z Galaxies with WFC3 Pure Parallel / HST Proposal 11702", http://archive.stsci.edu/cgi-bin/proposal_search?mission=hst\&id=11702

[48] Schneider, D. P., Schmidt, M. \& Gunn, J. E., "PC $1247+3406$ : An optically selected quasar with a redshift of 4.897', Astron. J. 102, 837-840 (1991). 
[49] Fan, X. et al., "A survey of $z>5.8$ quasars in the Sloan Digital Sky Survey. I. Discovery of three new quasars and the spatial density of luminous quasars at z 6", Astron. J. 122, 2833-2849 (2001).

[50] Becker, R. H. et al., "Evidence for re-ionization at $\mathrm{z} \sim 6$ : Detection of a Gunn-Peterson trough in a $\mathrm{z}=6.28$ quasar", Astron. J. 122, 2850-2857 (2001).

[51] Fan, X. et al., "Evolution of the ionizing background and the epoch of re-ionization from the spectra of $\mathrm{z} \sim 6$ quasars", Astron. J. 123, 1247-1257 (2002).

[52] Fan, X. et al., "A survey of $z>5.7$ quasars in the Sloan Digital Sky Survey. II. Discovery of three additional quasars at $\mathrm{z}>6$ ", Astron. J. 125, 1649-1659 (2003).

[53] Fan, X. et al., "Constraining the evolution of the ionizing background and the epoch of re-ionization with $\mathrm{z} \sim 6$ quasars II: A sample of 19 quasars", ArXiv Astrophysics e-prints <astro-ph/0512082> (2006).

[54] Gunn, J. E. \& Peterson, B. A., "On the density of neutral hydrogen in intergalactic space”, Astrophys. J. 142, 16331641 (1965).

[55] Kawai, N. et al., "Afterglow spectrum of a gamma-ray burst with the highest known redshift z $=6.295$ ”, Nature, 440, 184-186 (2006).

[56] Totani, T. et al., "Implications for cosmic re-ionization from optical afterglow spectrum of the Gamma-Ray Burst 050904 at z $=6.3$ ", PASJ, 58, 485, (2006)

[57] Tanvir, N. R.\& Jakobsson, P., “Observations of GRBs at high redshift”, astro-ph/0701777 (2007)

[58] Miyazaki, S. et al., "Subaru prime focus camera: Suprime-Cam”, Publ. Astron. Soc. Jpn 54, 833-853 (2002).

[59] Dunkley, J. et al., "Five-year Wilkinson Microwave Anisotropy Probe (WMAP) Observations: Likelihoods and Parameters from the WMAP data", arXiv:0803.0586v1, (2008)

[60] Dijkstra,M. and Wyithe, S.B., "Very massive star in high-redshift galaxies", Mon. Not. R. Astron. Soc., 379, 1589$1598(2007)$

[61] Gott, III, J.R., "Implications of the Copernican principle for our future prospects”, Nature, 363, 315-319 (1993) 